\title{
Investigation of the dorsolateral branch of the posterior intercostal artery for use as the pedicle of a free flap: A cadaveric study and case series
}

\author{
Su Bong Nam ${ }^{1}$, Jung Yeol Seo ${ }^{1}$, Tae Seo Park ${ }^{1}$, Ji Yoon Sung ${ }^{1}$, Joo Hyoung Kim ${ }^{1}$, Jae Woo Lee ${ }^{1}$, \\ Min Wook Kim ${ }^{1}$, Heung Chan $\mathrm{Oh}^{2}$ \\ ${ }^{I}$ Department of Plastic and Reconstructive Surgery, Pusan National University School of Medicine, Busan; ${ }^{2} U P$ Plastic Surgery Clinic, Busan, \\ Korea
}

Background The dorsolateral branch of the posterior intercostal artery (DLBPI) can be easily found while harvesting a latissimus dorsi (LD) musculocutaneous flap for breast reconstruction. However, it remains unknown whether this branch can be used for a free flap and whether this branch alone can provide perfusion to the skin. We examined whether the DLB$\mathrm{PI}$ could be reliably found and whether it could provide sufficient perfusion.

Methods We dissected 10 fresh cadavers and counted DLBPIs with a diameter larger than 2 $\mathrm{mm}$. For each DLBPI, the following parameters were measured: distance from the lateral margin of the LD muscle, level of the intercostal space, distance from the spinal process, and distance from the inferior angle of the scapula.

Results The DLBPI was easily found in all cadavers and was reliably located in the specified area. The average number of DLBPIs was 1.65. They were located between the seventh and eleventh intercostal spaces. The average length of the DLBPI between the intercostal space and the LD muscle was $4.82 \mathrm{~cm}$. To assess the perfusion of the DLBPIs, a lead oxide mixture was injected through the branch and observed using X-rays, and it showed good perfusion.

Conclusions The DLBPI can be used as a pedicle in free flaps for small defects. DLBPI flaps have some limitations, such as a short pedicle. However, an advantage of this branch is that it can be reliably located through simple dissection. For women, it has the advantage of concealing the donor scar underneath the bra band.

Keywords Surgical flaps / Free tissue flaps / Reconstructive surgical procedures / Cadaver
Correspondence: Heung Chan Oh UP Plastic Surgery Clinic, 75 Sesil-ro, Haeundae-gu, Busan 48106, Korea Tel: +82-55-360-1439 Fax: +82-55-701-2121

E-mail: upplasticsurgery@gmail.com
This work was supported by a 2-year research grant of Pusan National University.

This study was presented in part at the First Research and Reconstructive Forum, Congress of the Korean Society of Plastic and Reconstructive Surgeons, Daegu, Korea, May 09, 2013.

Received: 22 Nov $2017 \bullet$ Revised: 7 Sep $2018 \bullet$ Accepted: 2 Oct 2018

pISSN: 2234-6163 • elSSN: 2234-6171 • https://doi.org/10.5999/aps.2018.00122 • Arch Plast Surg 2019;46:39-45

\section{INTRODUCTION}

Some vessels with a large diameter are easily found when raising a latissimus dorsi (LD) flap for procedures such as breast reconstructive surgery (Fig. 1). These vessels include the dorsolateral branch of the posterior intercostal artery (DLBPI). The DLBPI appears to be large enough to be used as a pedicle of a free flap, which is a compelling possibility because doing so would completely conceal the donor scar under the bra band, unlike the thoracodorsal artery perforator (T-DAP). This would be advan-

Copyright (C) 2019 The Korean Society of Plastic and Reconstructive Surgeons

This is an Open Access article distributed under the terms of the Creative Commons Attribution Non-Commercial License (http://creativecommons.org/

licenses/by-nc/4.0/) which permits unrestricted non-commercial use, distribution, and reproduction in any medium, provided the original work is properly cited.

www.e-aps.org 


\section{Fig. 1. DLBPI and schematic diagram}

Dissection of the dorsolateral branch of the posterior intercostal artery (DLBPI) during a latissimus dorsi (LD) musculocutaneous flap operation for breast reconstruction. Lateral border, lateral margin of the LD muscle; inferior border, 12th rib; medial border, spinal processes; superior border, inferior angle of the scapula.

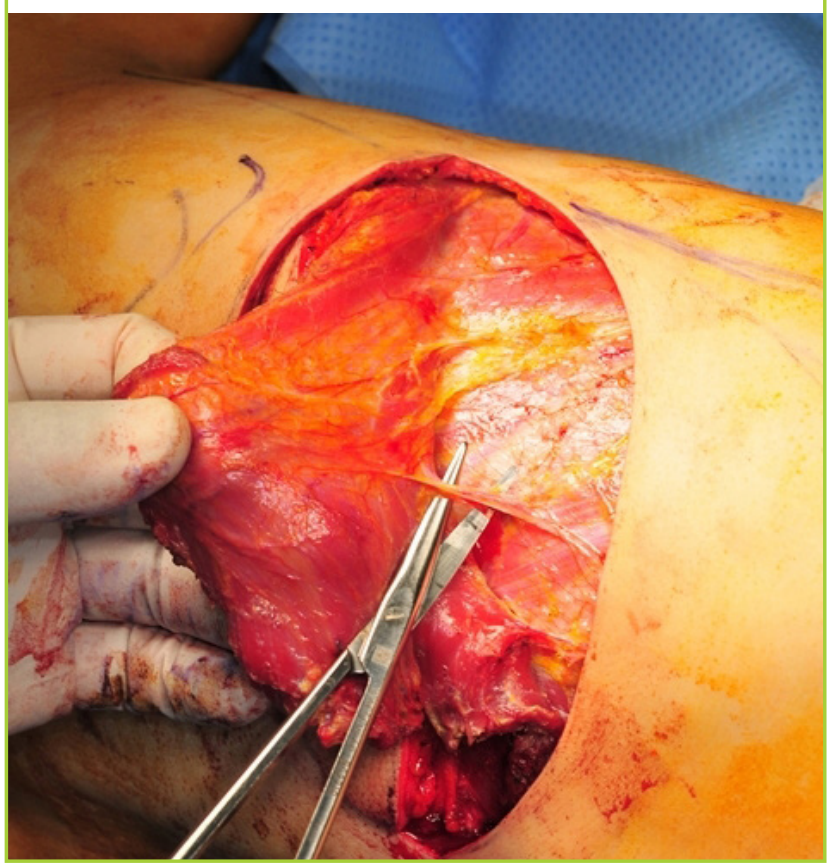

tageous for patients' quality of life, but no report has yet described the use of this branch as the pedicle of a free flap.

The location of the scar at the donor site after breast reconstruction is a critical issue for women. Thus, reports have described flap harvesting designs that can hide the donor site underneath the bra band [1-3]. The authors of the present study employ such designs for breast reconstruction and have observed that patients tend to exhibit interest in the location of the eventual scar tissue.

Pedicled island flaps using the dorsal branch of the posterior intercostal artery were described in a study that analyzed intercostal artery perforator flaps [4]. However, the dorsolateral branch was not clearly distinguished from the dorsal branch of the posterior intercostal artery in that study. Subsequent anatomical research has yielded more information about the DLBPI, including its location and origin [5]. However, to our knowledge, it remains unknown whether this branch can be used as the pedicle of a free flap and whether this branch alone can provide perfusion to the regional skin.

The purpose of this study was to clarify the anatomy of the DLBPI through a cadaveric study and to assess the usability of the flap through the results obtained when applying it in clinical settings.

\section{METHODS}

\section{Anatomical study by cadaver dissection}

We dissected 10 cadavers: eight that had been fresh-frozen and two embalmed cadavers. Five cadavers were male and five were female. A total of 20 flaps were elevated. Six cadavers were dissected in the prone position, while four were dissected in the lateral decubitus position, which is used for the surgical procedure, in order to determine whether the location of the perforator changed in realistic surgical conditions.

The flap was marked using the following landmarks. The lateral margin of the LD muscle was used as the lateral border, the 12th rib was used as the inferior border, a line $2 \mathrm{~cm}$ lateral from the spinal process was used as the medial border, and the inferior angle of the scapula was used as the superior border. The LD muscle was identified by incising the design down to the subcutaneous layer with a No. 10 scalpel blade. Initially, the lateral border of the LD muscle was identified. The muscle was incised along the inferior border of the rib by palpating the thoracic cage, and then incised upward along the line drawn laterally from the spinal process. Finally, after making a horizontal incision at the inferior angle of the scapula, the upper border of the LD muscle was identified. The LD muscle was then divided from the teres major and trapezius muscles. The DLBPI was subsequently identified as the inner surface of the LD muscle was dissected upward.

Only DLBPIs with an external diameter larger than $2 \mathrm{~mm}$ were counted, as smaller branches were not considered to be useful in actual clinical practice. For each branch, the following parameters were measured: distance from the lateral margin of the $\mathrm{LD}$ muscle, level of the intercostal space, distance from the spinal process, and distance from the inferior angle of the scapula. The length of the branch was estimated by measuring from the intercostal space to the inner surface of the LD muscle (Fig. 2).

To investigate the extent to which the DLBPI was capable of perfusing the flap, a 24-gauge catheter was inserted into the branch of an LD musculocutaneous (MC) flap harvested from a fresh cadaver. A mixture of lead oxide, milk powder, and tap water was then injected, as in the study carried out by Pan et al. [6]. The area that was supplied with a contrast dye from the perforator was then identified using X-ray mammography (Digital mammography; Siemens Medical Solutions, Forchheim, Germany). The X-ray examination was repeated after removing the LD muscle and leaving only the skin layer, as the dye that spread throughout the LD muscle and onto the skin layer appeared to overlap in the initial images. 


\section{Fig. 2. View of the cadaveric dissection}

Dissection of the dorsolateral branch of posterior intercostal artery in a cadaver and measuring the length of the pedicle in a cadaver.

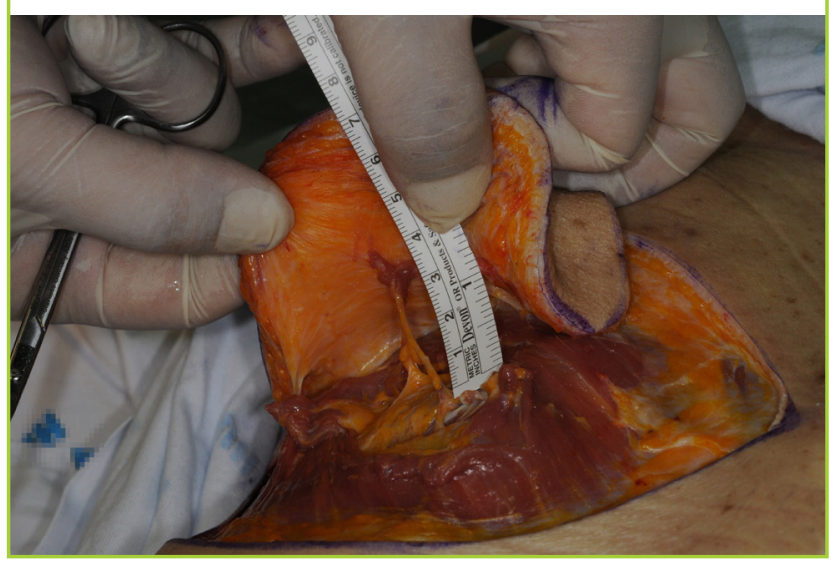

\section{Clinical trial}

Based on the above anatomic study, an LD MC flap with the DLBPI as its pedicle was harvested in one patient with soft tissue defects in the face and in two patients with defects in the lower extremity. Based on the preoperative scapular tip, the area extending from the seventh to eleventh intercostal spaces was observed, and based on the anatomical results from the spine and scapular tip, the location of the DLBPI was identified using Doppler ultrasonography and then marked. A chest computed tomography (CT) fine-section scan was obtained, and the location of the perforator identified through Doppler ultrasonography was compared to ensure the presence of the DLBPI (Fig. 3). During intraoperative flap elevation, the flap was designed to be centered on the perforator, and the perforator was observed from the submuscular dissection through subcutaneous incision and muscle stripping.

\section{RESULTS}

\section{Anatomical study by cadaver dissection}

Dissection of the cadavers revealed that the average number of DLBPIs (diameter $\geq 2 \mathrm{~mm}$ ) was $1.65 \pm 0.67$, and that they were located between the 7th and 11th intercostal spaces. On average, the branches were located $9.79 \pm 2.04 \mathrm{~cm}$ lateral from the spinal process, $9.18 \pm 2.06 \mathrm{~cm}$ inferior from the inferior angle of the scapula, and $9.79 \pm 2.32 \mathrm{~cm}$ medial from the lateral border of the LD. The average length of the branch between the intercostal space and the LD muscle was $4.82 \pm 1.07 \mathrm{~cm}$ (Table 1). The DLBPI was easily found in all cadavers dissected in the study and was reliably located in the specified area.

To assess the perfusion of DLBPI, a lead oxide mixture was injected, and the extent of the spread of the dye was observed.

\section{Fig. 3. DLBPI in a patient}

The location of the dorsolateral branch of the posterior intercostal artery (DLBPI) in an actual patient is identical to that observed in the anatomical study; on chest computed tomography (fine section, $1 \mathrm{~mm}$ ), the DLBPI was observed in the same location as was marked on Doppler ultrasonography (white arrow: DLBPI).

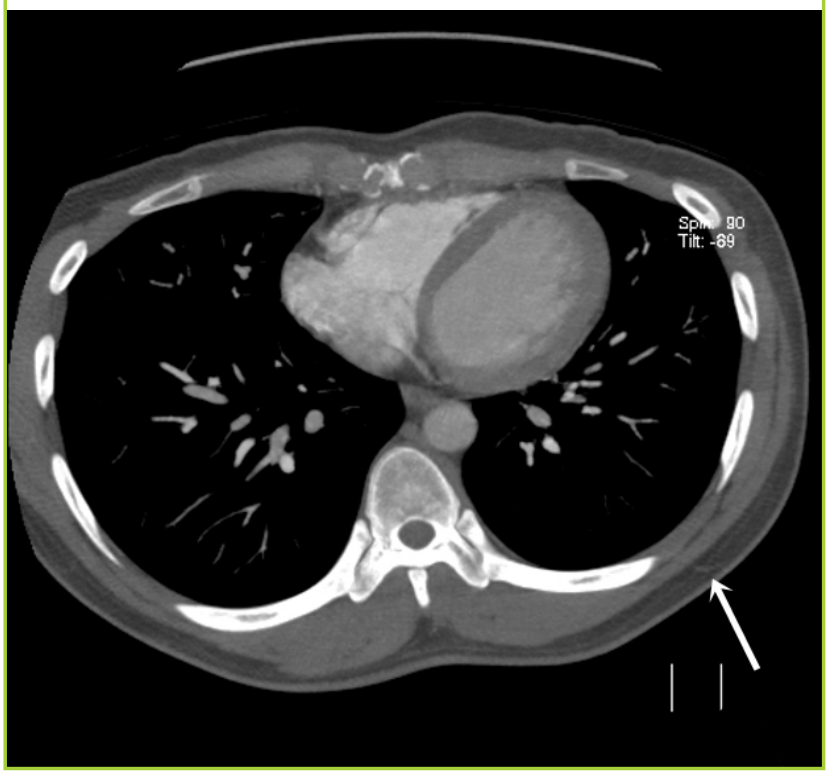

Table 1. Data regarding the DLBPI from 10 cadavers

\begin{tabular}{|lll|}
\hline Data of measurement & Range & \multicolumn{1}{c|}{ Mean } \\
\hline Intercostal space & $7-11$ th & $8.61 \pm 0.97$ th \\
No. of DLBPIs (diameter $\geq 2 \mathrm{~mm})$ & $1-3$ & $1.65 \pm 0.67$ \\
Distance from the spinal process (cm) & $7-16$ & $9.79 \pm 2.04$ \\
Distance from the inferior angle of the scapula (cm) & $6-13$ & $9.18 \pm 2.06$ \\
Distance from the lateral border of the LD (cm) & $4-14$ & $9.79 \pm 2.32$ \\
Length of the pedicle (cm) & $3-7$ & $4.82 \pm 1.07$ \\
\hline DLBPI, dorsolateral branch of posterior intercostal artery; LD, latissimus dorsi. \\
\hline
\end{tabular}

However, contrary to our expectations, when injected, the dye spread over the entire area of the elevated flap. An X-ray scan of the site was then performed. The range of the LD muscle was consistent with the extent of the dye, confirming good perfusion in the LD MC flap (Fig. 4A). Nonetheless, the extent of the dye in the skin layer and the muscle layer overlapped. Therefore, the $\mathrm{X}$-ray scan was repeated after removing the $\mathrm{LD}$ muscle in order to determine whether the DLBPI provided sufficient perfusion to the skin layer. We found that the skin layer was well perfused (Fig. 4B).

\section{Clinical trial}

\section{Case 1}

For the reconstruction of a soft tissue defect in the left infraorbital region that occurred after the excision of maxillary cancer in a male patient, an MC free flap $(12 \times 5 \mathrm{~cm})$ with a DLBPI in 


\section{Fig. 4. X-ray (mammography) findings of perfusion}

(A) X-ray (mammography) of a latissimus dorsi (LD) musculocutaneous flap after injection of a lead oxide mixture into the dorsolateral branch of the posterior intercostal artery. (B) Repeated X-ray (mammography) after removing the LD muscle, with only the skin layer included.
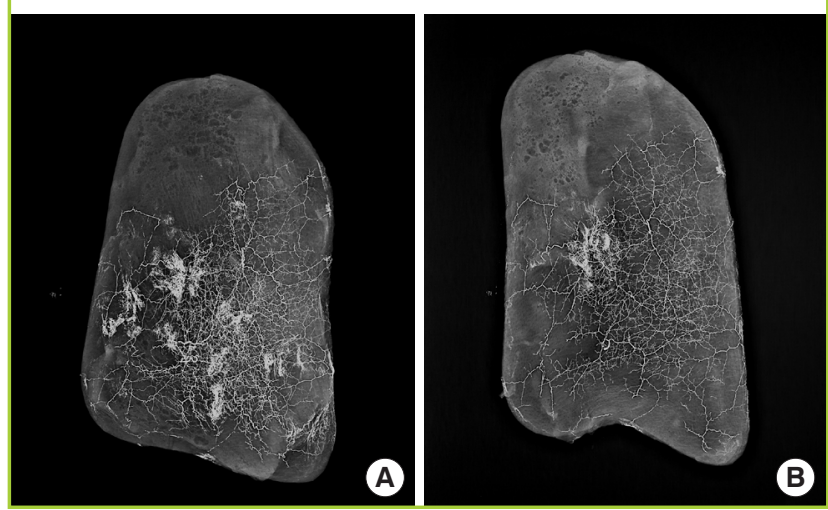

the left side of the patient's back was harvested and then anastomosis was done to the angular artery to compensate for the defect. At this time, to extend the length of the pedicle, the external intercostal muscle and rib bone were partially excised (Fig. 5).

\section{Case 2}

In a female patient with soft tissue defects in the left lower leg caused by trauma, a free MC flap $(15 \times 7 \mathrm{~cm})$ was harvested with the DLBPI from the left side of the patient's back (Fig. 6). The recipient vessel was the branch of posterior tibial artery and vena comitans.

\section{Case 3}

In a female patient with a soft tissue defect in the right foot caused by diabetes, reconstruction in the form of a free MC flap $(12 \times 4 \mathrm{~cm})$ with the DLBPI from the left side of the patient's back was performed. The recipient vessel was the plantar metatarsal artery and vena comitans (Fig. 7).

\section{DISCUSSION}

In 1987, Taylor [7] reported that detecting angiosomes plays a pivotal role in identifying perforator flaps. They also demonstrated that vessels supplying the skin can be used to create a flap. Angiographic studies have identified angiosomes and proven that various body parts can be used as donor sites for free skin transfer, such as in the form of a perforator free flap [8]. Pedicled perforator flaps have been widely used, and perforator flaps are used for free skin transfer.

To use a free flap with the DLBPI, the authors thought that an anatomical study would be necessary and accordingly used 10

\section{Fig. 5. Case 1}

For the reconstruction of a soft tissue defect in the left infraorbital region that occurred after the excision of maxillary cancer in a male patient, a musculocutaneous $(\mathrm{MC})$ free flap $(12 \times 5 \mathrm{~cm})$ with a dorsolateral branch of the posterior intercostal artery (DLBPI) in the left side of the patient's back was harvested. (A) Preoperative view. (B) Elevation of the DLBPI MC flap. (C) Immediate postoperative view. (D) Immediate postoperative view after defatting of the flap.
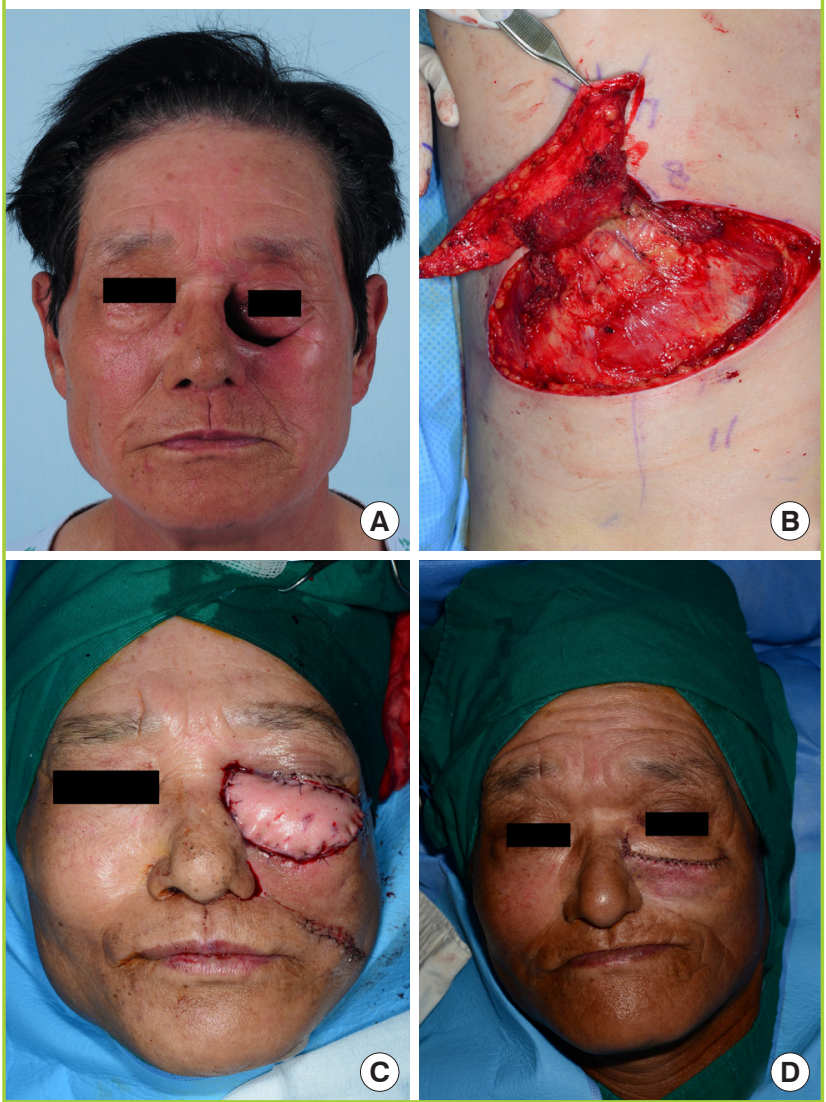

cadavers to check the location and distribution of the DLBPI. Offman et al. [9] reported that the perforator from the posterior intercostal artery was variable, but in this study, all perforators could be observed from the seventh to eleventh intercostal spaces. Thus, it was possible to find a pedicle measuring $9.18 \mathrm{~cm}$ on average from the scapular tip, $9.79 \mathrm{~cm}$ on average from the spinal process, and $4.82 \mathrm{~cm}$ on average laterally. Despite differences between Western and Asian populations, this finding was similar to the result reported by Prasad et al. [5] that the perforator had a pedicle measuring an average of $4.6 \mathrm{~cm}$ in the eight to eleventh intercostal spaces. In our study, dissection of the cadavers revealed that the average number of DLBPIs (diameter $\geq 2$ $\mathrm{mm}$ ) was $1.65 \pm 0.67$, and that they were located between the 7 th and 11th intercostal spaces. The DLBPI was easily found in all cadavers dissected in the study and was reliably located in the specified area.

In the cadaveric study, we investigated the extent to which the 


\section{Fig. 6. Case 2}

In a female patient with soft tissue defects in the left lower leg caused by trauma, a free musculocutaneous (MC) flap $(15 \times 7 \mathrm{~cm})$ was harvested with a dorsolateral branch of the posterior intercostal artery (DLBPI) from the left side of the patient's back. (A) Preoperative view. (B) Preoperative design of the flap with marking of DLBPI. (C) Elevation of the DLBPI MC flap. (D) Immediate postoperative view. (E) Postoperative view (19 months postoperatively). After removal of areas of necrotic change in the flap, a skin graft was performed. However, a pressure sore on the lateral malleolar region occurred.
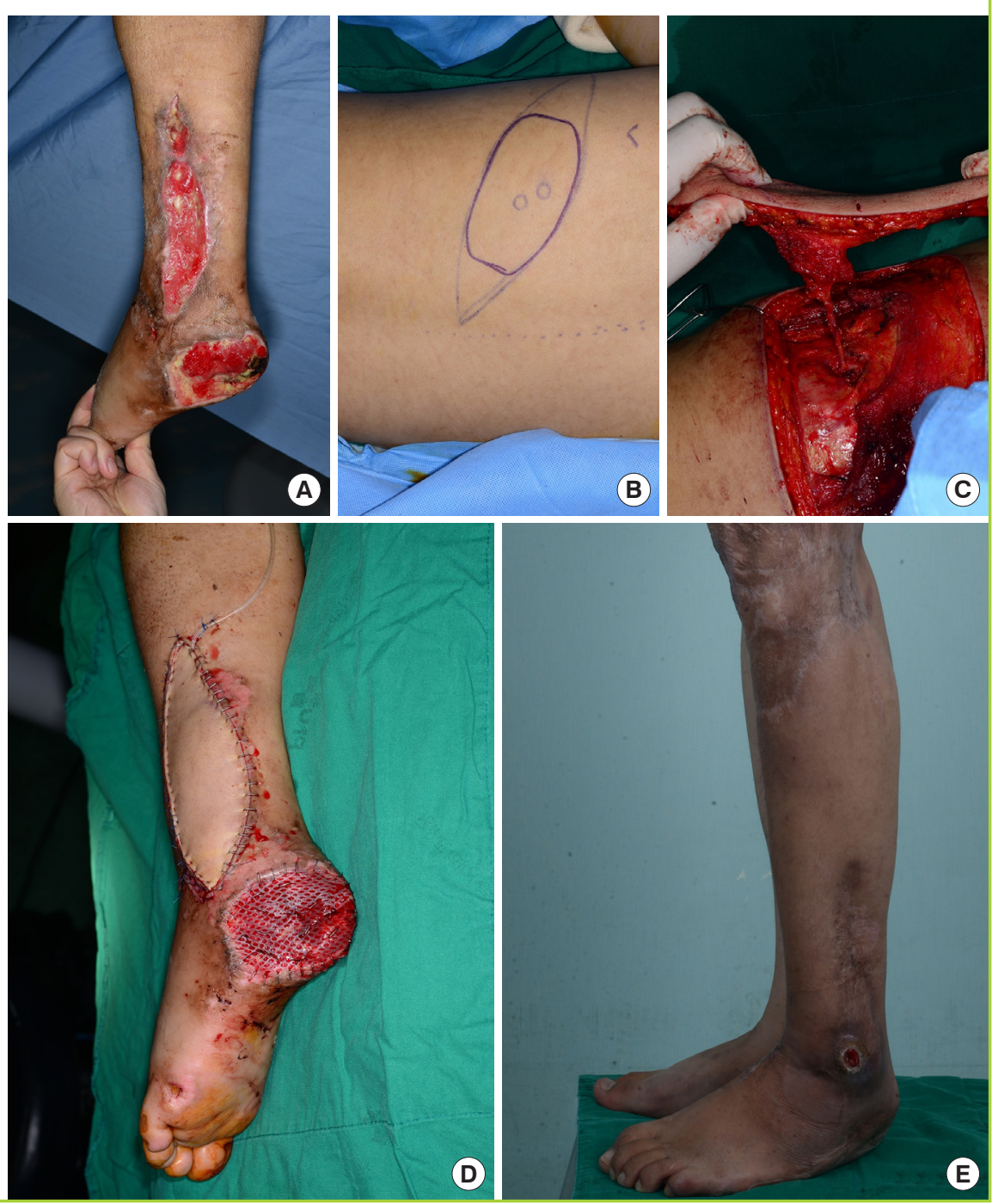

dye spread using mammography, which involves a greater dose of radiation than ordinary X-ray scans. Our initial speculation was that when the dye was injected into the DLBPI, it would spread throughout a limited area, which would show the perfusion capability of the branch. Concerns were raised that the injection pressure, the viscosity of the dye, and the concentration of the dye could act as confounding factors affecting the extent to which the dye would spread. However, when the injection was made, the dye diffused throughout the system easily. Immediately after the injection, the dye spread throughout the entire system, overflowing the margins. Based on this observation, the vessel network around this area appears to be very well developed. Nonetheless, perfusion to the skin layer is presumed to be supplied through the muscle layer for the following reasons: the dye spread throughout the area corresponding to the LD muscle both when the MC flap was present and when only the skin lay- er was imaged, and countless small vessels between the skin and muscle were observed when the LD muscle was removed from the skin layer.

Based on these anatomical results, it was concluded that the location of the perforator could be found using Doppler ultrasonography preoperatively, with the patient in the sitting position, and the perforator was confirmed through chest CT (1-mm fine sections). Therefore, it was deemed possible to design the DLBPI flap using a pinch test centered on the location of the perforator determined by Doppler ultrasonography. Subsequently, reconstruction with this flap was conducted in patients with defects, who were considered suitable for anastomosis with a short pedicle in various areas.

Several considerations must be taken into account when performing a free flap with the DLBPI in clinical settings. First, the pedicle of the DLBPI free flap is short $(4-5 \mathrm{~cm})$. Therefore, the 


\section{Fig. 7. Case 3}

In a female patient with a soft tissue defect in the right foot caused by diabetes, reconstruction in the form of a free musculocutaneous (MC flap, $12 \times 4 \mathrm{~cm}$ ) with a dorsolateral branch of the posterior intercostal artery (DLBPI) from the left side of the patient's back was performed. (A) Intraoperative view after debridement. (B) Preoperative design of flap with marking of the DLBPI. (C) Elevation of the DLBPI MC flap. (D) Intraoperative view after perforator-to-perforator anastomosis.
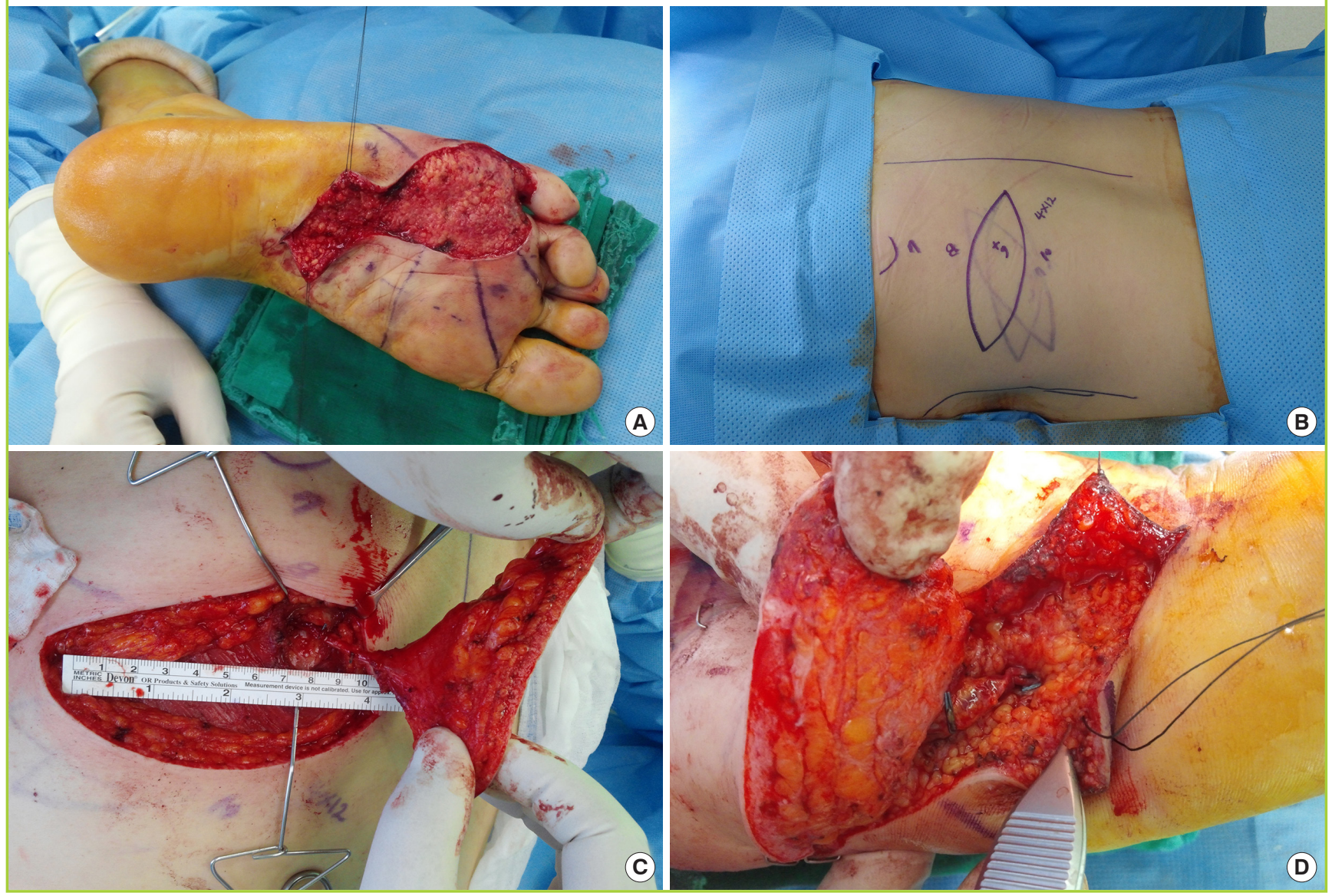

recipient vessel must be located close to the defect. Moreover, if the condition of the recipient vessel at the time of anastomosis is not optimal, the procedure cannot be performed successfully. The DLBPI flap for lower extremity reconstruction involved perforator-to-perforator anastomosis, but anastomotic failure occurred due to a spasm of the recipient's vein (case 2) or artery (case 3). In case 2, the flap showed total necrosis at 10 days postoperatively, after which a secondary operation with a skin graft was required. In case 3 , the recipient arterial spasm was not corrected in 10 hours despite several efforts. Thus, we had to use the DLBPI flap as a full-thickness skin graft. Second, in patients with a thick subcutaneous fat layer, the flap was too thick, making it burdensome to use this flap (case 1). Third, it has been reported that elevating the flap by dissecting the posterior intercostal artery medially or laterally along the lower costal margin allowed the pedicle to be elongated to as long as $12 \mathrm{~cm}$ [5]. However, the intercostal muscle would be injured in such a procedure, which would become excessively complex, and the pedicle would be likely to be damaged (as the pedicle has many branches in the muscle layer), potentially causing adverse events such as spasms. Therefore, for extension, it is considered much easier to use another free flap (e.g., an anterolateral thigh flap) (case 1). Fourth, the DLBPI may be branched when it inserts into the LD muscle. Such cases require meticulous care, and further dissection slightly deep to the origin in the intercostal muscle can be helpful. Fifth, the DLBPI free flap is safer for use as an MC flap. As previously discussed, it is speculated to be perfused via the $\mathrm{LD}$ muscle layer. Therefore, unlike the T-DAP flap or the anterolateral thigh flap, this flap is risky if much of the muscle layer is removed to skeletonize the pedicle or in an attempt to make the flap thinner. Finally, the patient must be placed in the lateral decubitus position during surgery for flap harvesting.

This study investigated the possible use of the DLBPI as the perforator of a free flap. Despite its limitations, the DLBPI free flap may be considered as an alternative free flap for small de- 
fects. The major advantage of the DLBPI free flap is that harvesting it is easy and simple, because the DLBPI can be easily found in a reliable location, with rare anatomical variants. Additionally, for women, it has the advantage of concealing the donor scar underneath the bra band, which is beneficial for patients' quality of life.

\section{NOTES}

\section{Conflict of interest}

No potential conflict of interest relevant to this article was reported.

\section{Ethical approval}

The study was approved by the Institutional Review Board of Pusan National University Yangsan Hospital (IRB No. 05-2018137) and performed in accordance with the principles of the Declaration of Helsinki. Written informed consent was obtained.

\section{Patient consent}

The patients provided written informed consent for the publication and the use of their images.

\section{ORCID}

Su Bong Nam https://orcid.org/0000-0002-9661-0879

Jung Yeol Seo https://orcid.org/0000-0003-0715-2229

Heung Chan Oh https://orcid.org/0000-0003-3209-6310

\section{REFERENCES}

1. Fatah F. Extended latissimus dorsi flap in breast reconstruction. Oper Tech Plast Reconstr Surg 1999;6;38-49.

2. Menke H, Erkens M, Olbrisch RR. Evolving concepts in breast reconstruction with latissimus dorsi flaps: results and follow-up of 121 consecutive patients. Ann Plast Surg 2001; 47:107-14.

3. Chang DW, Youssef A, Cha S, et al. Autologous breast reconstruction with the extended latissimus dorsi flap. Plast Reconstr Surg 2002;110:751-9.

4. Hamdi M, Van Landuyt K, de Frene B, et al. The versatility of the inter-costal artery perforator (ICAP) flaps. J Plast Reconstr Aesthet Surg 2006;59:644-52.

5. Prasad V, Almutairi K, Kimble FW, et al. Dorsolateral musculocutaneous perforators of posterior intercostal artery: an anatomical study. J Plast Reconstr Aesthet Surg 2012;65: 1518-24.

6. Pan WR, Cheng NM, Vally F. A modified lead oxide cadaveric injection technique for embalmed contrast radiography. Plast Reconstr Surg 2010;125:261e-262e.

7. Taylor GI. The angiosomes of the body and their supply to perforator flaps. Clin Plast Surg 2003;30:331-42.

8. Taylor GI, Daniel RK. The anatomy of several free flap donor sites. Plast Reconstr Surg 1975;56:243-53.

9. Offman SL, Geddes CR, Tang M, et al. The vascular basis of perforator flaps based on the source arteries of the lateral lumbar region. Plast Reconstr Surg 2005;115:1651-9. 